\title{
Mismanagement of Antibiotics in Neonatal Medicine
}

\author{
Marcelo Cardetti ${ }^{1}$, Susana Rodríguez ${ }^{2}$, and Augusto Sola ${ }^{3 *}$ \\ ${ }^{1}$ Neonatology, Center for Endocrinology and Human Reproduction and Ibero American Society of Neonatology (SIBEN), Argentina \\ ${ }^{2}$ Education and Research, Garrahan Hospital and Ibero American Society of Neonatology (SIBEN), Argentina
}

${ }^{3}$ Ibero American Society of Neonatology (SIBEN), USA

*Corresponding author: Augusto Sola, Medical Director, Ibero American Society of Neonatology (SIBEN), 2244 Newbury Dr, Wellington, Fl, USA.

Received Date: March 10, 2020

Published Date: March 17, 2020

\begin{abstract}
Unreasonable use of antibiotics occurs in about $25-30 \%$ of the population. The neonatal period is among the highest group where antibiotic abuse occurs, as high as $40 \%$ in some hospitals. This is particularly apparent in newborn intensive care units (NICU), where $70-80 \%$ of the admitted infants receive antibiotics. The main motive for this is that clinicians suspect neonatal sepsis very frequently, but only about 3-5\% or less of the time infants have blood culture proven sepsis. The neonatal sepsis calculator, described some years ago and validated by several studies, is extremely useful in allowing care givers to assess risk factors and combine them with the clinical evaluation of the newborn to make a more adequate decision and decrease utilization of unnecessary antibiotics. On the other hand, nonspecific inflammatory markers, like C-reactive protein (CRP) and procalcitonin (PCT), are relied upon to make clinical decisions about antibiotic initiation and discontinuation. However, they have very bad specificity for early and late neonatal sepsis, and their sensitivity is not very adequate either. Relying on them in suspicion of neonatal sepsis is a "hazardous waste". Antibiotic abuse is associated with short- and long-term adverse effects. In preterm infants in NICU, length of hospital stay, bronchopulmonary dysplasia, necrotizing enterocolitis and mortality are increased. In addition to this and to the concomitant increase in health care expenditures, there are long lasting consequences of antibiotic misuse in the neonatal period. They include development of antibiotic resistance, with the emergence of multi resistant organisms, and alterations to the microbiota and microbiome. This has been linked to various disease states later in life, such as abnormal brain development, infections during childhood, asthma, obesity, diabetes, atherosclerosis and autoimmune disorders, among others. The need to apply clinical measures to modify current neonatal practices and improve outcomes has never been more urgent.
\end{abstract}

Keywords: Antibiotics; Abuse; Newborn; Neonatal; Microbiome

Abbreviations: NICU: Newborn intensive care unit; CRP: C-reactive protein; PCT: Procalcitonin; EOBS: Early onset bacterial sepsis; WHO: World Health Organization; NEC: Necrotizing enterocolitis; BPD: Bronchopulmonary dysplasia; AMR: Antimicrobial resistance; NNT: Number needed to treat; SIBEN: Ibero american society of neonatology

\section{Introduction}

"The rational use of drugs requires that patients receive medications appropriate to their clinical needs, in doses that meet their individual requirements, for an adequate period of time, and at the lowest cost to them and their community" was affirmed by the Conference of Experts on the Rational Use of Drugs convened by the World Health Organization (WHO) in Nairobi in 1985 [1]. WHO estimates that more than half of all medicines are prescribed inappropriately, and antibiotics are among the most frequent ones that are used incorrectly [2]. Furthermore, it has become apparent that newborn infants are exposed to the highest abuse rate of antibiotics. For example, more than to $70 \%$ of neonates admitted to some neonatal intensive care units (NICU's) are prescribed antibiotics [3,4]. In the USA, data from 297 centers with 40,364 infants $<1,500$ grams [5] showed that the majority of infants born prematurely infants are exposed to early antibiotic initiation and this occurred in $78.6 \%$ of infants with birth weight $<1,500$ grams and in $87 \%$ of those $<1,000$ grams at birth. Furthermore, many of them had subsequent prolonged antibiotic administration for more than 5 days. However, extremely few babies (1-3\%) with suspected neonatal early-onset bacterial sepsis (EOBS) are actually proven to have blood culture positive sepsis. 
In this manuscript we focus on the irrational uses of antibiotics in the neonatal period, particularly when EOBS is suspected. Clinical indications for initiation and discontinuation of antibiotics are described, together with the potential severe consequences of antibiotic abuse. These include, among others, higher prevalence of bronchopulmonary dysplasia (BPD), necrotizing enterocolitis (NEC) and mortality, in addition to development of antimicrobial resistance (AMR) and microbiome alterations. AMR and the emergence of multiresistant bacteria is an epidemic in public health. Neonatal microbiome influences immune cell ontogeny [6] and early exposure to antibiotics affect the composition of the intestinal microbiota [7-11].

The purpose of this manuscript is to review some of the current neonatal practices related to antibiotic indication and discontinuation and present a current summary of most of the untoward effects of antibiotic abuse in neonatal medicine. Hopefully, this review will be useful for implementation of changes that would ultimately lead to improved neonatal outcomes.

\section{Discussion}

\section{Use of antibiotics in the neonatal period}

Neonatal bacterial sepsis is a grave condition that may lead to death if untreated or if initiation of treatment is delayed. Antibiotics do save newborns' lives.

In the NICU, suspected EOBS is one of the most common diagnoses and antibiotics are the most frequently used medications. The antibiotics for treatment of neonatal sepsis must be given intravenously and never PO or intramuscularly. The more frequently used are ampicillin and gentamycin. Their discontinuation should occur in 36-72 hours if the blood cultures are negative and the infant is asymptomatic.

In asymptomatic newborns, with suspected EOBS decisions to start antibiotics should be mainly based on the newborn's clinical condition and not solely on risks or on inflammatory markers.

The risk of EOBS is very low in well appearing, asymptomatic newborns, about $0.21 \%$ live births [12-14]. The number needed to treat (NNT) for this group has been estimated at 9,370. What needs to be done in these cases is clinical assessment and risk assessment, with routine observation of the infants together with their mothers, and discharge home as per usual guidelines. On the other hand, when there are clinically evident signs in the first 12-24 hours of life, the risk is $>11 \%$ live births, with NNT of about 118 . In these cases, a blood culture and empirical antibiotics should be started immediately without delay, with admission to the NICU. In cases of equivocal or doubtful clinical early presentation, the risk is about $2.6 \%$ live births, with NNT of 823 . In these doubtful cases a blood culture can be obtained, and the vital signs must be carefully monitored every 4 hours for 24 hours at least, without initiation of antibiotics. Treatment with antibiotics will be according to the persistence of suggestive symptoms.
Risk scores and clinical assessment should guide the practice of antibiotic utilization, and not simply suspecting EOBS. In a study conducted on 608.014 newborns > 34 weeks in 14 hospitals [15] it was possible to stratify the risk of neonatal sepsis according to several maternal and labor and delivery variables, together with the newborn's clinical presentation. Three groups were identified:

\section{Treat empirically with antibiotics: $4.1 \%$}

2. Study them, with frequent observation and conditional treatment: $11.1 \%$

\section{Only continuous observation: $84.8 \%$.}

The incidence of sepsis with positive blood cultures was $8.4 \%$, $1.2 \%$ and $0.11 \%$ respectively for groups 1,2 and 3 . This has led to a decrease in the use and time of antibiotics in the network of participating hospitals. These authors created the neonatal sepsis calculator, a software that calculates an individual risk or score in relation to some prenatal factors and the baby's clinical signs and evolution in the first 12 to 72 hours [16]. This neonatal sepsis calculator, validated by several studies, is extremely useful in allowing clinicians to assess risk factors and combine them with the clinical evaluation of the newborn to make a more adequate decision in clinical care [12-14,17-23]. In an asymptomatic infant without any risk, or even with some risks, appropriate care is to observe, evaluate and not interfere with the mother-child bonding, unless the a priori risk is high or very high. When in doubt, take blood cultures, do not start antibiotics but implement detailed clinical observation every 2-4 hours of the infant while she or he is with the mother. If something changes clinically, start antibiotics promptly. However, in symptomatic newborns, even if the risk score is low, obtain blood cultures and start antibiotics quickly. If the symptoms disappear after several hours and blood cultures are negative, antibiotics could be discontinued in 48-72 hours.

\section{Chorioamnionitis and prolonged rupture of the mem- branes}

Routine antibiotics had been initiated in many, if not all neonates exposed to chorioamnionitis. However, in mothers with clinical chorioamnionitis, the incidence of EOBS in neonates has been reported as low as $1 \%$ o to about $4-7 \%$ live births. In newborns > 35 weeks' gestation, only about $1.25 \%$ of the infants born to mothers with chorioamnionitis have positive cultures. The rate is higher in newborns $<35$ weeks ( $4.8 \%$ to $16.9 \%$ ). This is clear proof that routine antibiotics should not be initiated in all neonates exposed to chorioamnionitis. Doing so would mean treating hundreds of infants to find one that really has an infection. The NNT in the group of infants $>35$ weeks exposed to chorioamnionitis is from 80 to 210. Additionally, studying the placenta histologically does not add substantial information to decide to start treatment or to prolong the use of antibiotics. All this suggests that we should abandon the recommendation to treat with antibiotics asymptomatic newborns $>34$ weeks with a history of chorioamnionitis [24-29]. Even 
in symptomatic infants born to mothers with chorioamnionitis, the NNT in order to identify one infant with EOBS is high, 23 or greater. However, in the case preterm infants with maternal chorioamnionitis, the NNT is from 6 to 20; in this group it would be justified to obtain blood cultures and start antibiotics.

Similarly, there is no need to treat all infants exposed to prolonged rupture of the membranes. If an infant is $\geq 37$ weeks and is asymptomatic there is no need to start antibiotics even if there is prolonged rupture of membranes $>18$ hours. Newborns $\geq 37$ with signs of respiratory distress should be observed during their transition and in case of worsening or persistence beyond 6 hours of life, blood cultures should be obtained, and they should be treated with ampicillin and gentamycin until obtaining the results of the cultures. In risk situations like prolonged rupture of the membranes >18-24 hours, chorioamnionitis and/or maternal Group B streptococcus partially treated, if the infant remains clinically asymptomatic with negative blood cultures, it is not justified to continue antibiotics for much longer than 48 hours.

\section{Nonspecific inflammatory markers}

Clinicians have historically relied upon nonspecific inflammatory markers, like C-reactive protein (CRP) and procalcitonin (PCT) to make clinical decisions about antibiotic initiation and discontinuation. We will not extend much on the unfortunate impact that inflammatory markers (and others) had on antibiotic abuse in neonatology worldwide, but it has been known for over a decade that they are very inaccurate for predicting neonatal bacterial sepsis. Despite the existing evidence, many clinicians continue to do venous or arterial punctures to obtain blood to measure nonspecific inflammatory markers and base their clinical decisions on "a value without value" to start or to continue antibiotics unfoundedly. The recommendation is not to obtain these determinations in clinically asymptomatic infants, which is now clearly supported by two recent systematic reviews and meta-analysis. One from January 2019 [30] and the other one from February 2020, which included 22 studies previously published, clearly confirm that CRP has very bad specificity for neonatal sepsis, and its sensitivity is not very adequate either [31]. In the title of the editorial that accompanies this publication, the authors say that evaluating CRP in suspicion of neonatal sepsis is a "hazardous waste" [32]. CRP and PCT have more clinical value when they are normal, meaning that the likelihood of infection is very low. Accordingly, if antibiotics had been started and blood cultures are sterile and the nonspecific inflammatory markers, if measured, are normal, it is imperative to discontinue antibiotics in 36-72 hours, unless there is clear evidence of site-specific infection. Moreover, persistent cardiorespiratory instability is common in preterm infants $<1,500$ grams and many clinicians continue antibiotics "just in case", but this is not an indication by itself to use prolonged empirical antibiotics.

In summary, through the past two decades it has become clear that the systematic, immediate use of antibiotics in most neonatal situations is not justified [28,33]. Therefore, there is an imperative need for more judicious use of antibiotics in neonates, discouraging their systematic empiric use when sepsis is only a remote possibility and a protracted course when blood cultures are negative [34-38]. By not starting antibiotics unnecessarily mother infant bonding is not disrupted and by not prolonging antibiotic therapy for "culture-negative sepsis" many short- and long-term adverse events discussed below are prevented.

\section{Abuse of antibiotics during the neonatal period}

In a cross-sectional study of 326,845 live births in 2017 in California hospitals, the percent of newborns with antibiotic exposure varied considerably from $1.6 \%$ to $42.5 \%$. This is unexplained by proven bloodstream infection and it was not correlated to the percent of patient-days entailing antibiotic exposure [39]. In one publication from one NICU the mean duration of antibiotics without proven infection was $10.8 \pm 7.3$ days [40]. Even worse, the most commonly used antibiotics were carbapenems and vancomycin and not ampicillin and gentamycin.

In NICU's $>40 \%$ of admitted babies are given antibiotics and even up to $90 \%$ in some of them. However, few babies (1-3\%) with suspected EOBS will be proven to have sepsis and therefore antibiotics are abused or misused. Additionally, there is significant variability across different NICU's in early antibiotic utilization and in the proportion of infants administered prolonged antibiotics. In Canada, for example, the rate of antibiotic use for suspected sepsis for the whole network was just above $40 \%$, but it varied considerably from about $20 \%$ in the lowest end to about $70 \%$ in the highest [3]. In the network of the Ibero american society of neonatology (SIBEN), with 40 NICU's from 10 Latin-American countries, the variability of antibiotic utilization for $>3$ days in infants with negative blood cultures during 2018 was astonishing: from $10 \%$ to $92 \%$. In 5 NICU's, more than $60 \%$ of the neonates received such management, while in 4 NICU's this was done in $20 \%$ or less of the infants. In preterm infants $<1,500$ grams with negative blood cultures there was an association between excessive prolonged antibiotics and mortality [4].

Why does all the disarray described above happen is multifactorial and escapes a detailed analysis in this manuscript. Nevertheless, the use of antibiotics in the NICU should ideally be directed so precisely that only newborns with a proven infection would receive antibiotics and, even then, receive those antibiotics with the narrowest effective spectrum [34].

\section{Morbidities and mortality in NICU associated to antibi- otic abuse}

Prolonged (often unnecessary) exposure to antibiotics during the neonatal period have been associated with significant morbidities in preterm infants like: NEC, late onset sepsis, BPD, systemic candidiasis, retinopathy of prematurity, emergence of multi-resistant microorganisms, increased length of hospital stays, and also 
increased in mortality [41-44]. In SIBEN's network, mentioned before, there was an association between excessive prolonged antibiotics and mortality in infants $<1,500$ grams with negative blood cultures [4].

\section{Childhood infections}

Higher risks of infection-related hospitalization in the first 6 years of life have been reported in association with perinatal antibiotics [hazard ratio (HR) 1.18, 95\% confidence interval (CI) 1.171.19] [45].

\section{Intestinal microbiota and microbiome}

Almost half the cells and 1\% of the unique genes found in our bodies are human, the rest are from microbes, predominantly bacteria, fungi, and viruses. These microorganisms collectively form the human microbiota, with most colonizing the gut. It is estimated that there are nearly 30 trillion bacterial cells living in or on each human. Recent technological advances have allowed the genes of these microbes to be identified; the microbiome is all the genes that the microbiota contains. This "newly discovered organ" is a rapidly evolving, highly multidisciplinary field [46]. It is known that exposure to microbes first occurs during birth and is later influenced by environmental factors, such as diet and exposure to antibiotics , which affect the composition of the intestinal microbiota and microbiome [7-11,47]. Neonatal microbiome influences immune cell ontogeny and, as mentioned below, there is a link between perturbations in the gut microbiota and neurological disease $[6,46,48]$. Perinatal microbiome and early life immunity have a role in regulating normal neurodevelopmental processes and there is evidence that abnormalities or perturbations of the human microbiome during early life are associated with altered risk for neurological disorders and cancer development in humans [6]. Even though there is still much more to uncover, there is convincing evidence that they play a significant role in specific cancers. Interestingly, the normal microbiome can support the immune system in the fight against cancer [49].

Class of antibiotics relates to different maturation of infant gut microbiota [9] and may have long-term effects on the early microbial colonization of the newborn [10]. The roles for the perinatal microbiome are complex but interfering with the microbiome may delay or even obstruct the natural process of perinatal immune priming. Illnesses like autoimmune and allergic disorders, including childhood asthma, may be imprinted during infancy, only to manifest later in life [50]. In addition, perturbations in microbiota and microbiome during early life are also associated with obesity $[51,52]$.

Other conditions associated with disruption of the microbiome are celiac disease [53], Crohn's disease (7 times higher risk) [54,55], juvenile idiopathic arthritis [56] and, maybe, intestinal cancer [57].

In summary, the widespread use of neonatal antibiotics has raised concerns about the possible disruption of the child's gut mi- crobiota and the effects on microbiome maturation from infancy to adulthood. Even though the dose-dependent nature of the effect of narrow or broad-spectrum antibiotics, the number of antibiotics and the timing of their use that produce microbiome modifications have not yet been well defined [8], a single course of antibiotics perturbs bacterial communities that do not return completely to baseline following treatment, affecting the developmental trajectory of the infant gut microbiome $[9,10]$.

\section{Impact on the developing brain}

In addition to the above, emerging evidence links perturbations in the gut microbiota to neurological disease, including disease risk, activity, and progression [46,48]. Neonatal microbiome influences immune cell ontogeny. Perinatal microbiome and early life immunity have a role in regulating normal neurodevelopmental processes and there is evidence that abnormalities in microbiota-neuroimmune interactions during early life are associated with altered risk for neurological disorders in humans [6].

Prenatal exposure to antibiotics has been associated with an increased risk of epilepsy in childhood [58]. Among children exposed prenatally, the incidence rate was 117 per 100,000 person-years, with an adjusted ratio of 1.40 (95\% CI: 1.22-1.61), compared with unexposed children. The association was unaffected by trimester of exposure, antibiotic type, or prescription count [58]. It remains to be determined if prolonged exposure to antibiotics after birth increases this prevalence.

\section{Negative effects of cumulative exposure to antibiotics during development}

The necessity to treat symptomatic bacterial infections is clear, but it is also clear that during the last 20 years there has been an inappropriate overuse of antibiotics in neonatal medicine worldwide. Even though this happens across all gestational ages, in a cohort study from 13 centers of 5,730 preterm infants with birth weight 401-1000 grams and gestational age 22-28 weeks, prolonged early antibiotics (defined as $\geq 5$ days of antibiotic therapy started at $\leq$ 72 hours of age) without culture-confirmed infection or NEC , varied from 30-69\% among centers [59]. Based on what is known to date, one can only wonder what the negative impact could be for infants who are exposed to prolonged cumulative exposure to antibiotics during a crucial stage of development for days or weeks after birth, without any clear documentation of infection.

\section{Antimicrobial resistance (AMR)}

Misuse of antibiotics has resulted in the rapid rise of AMR and development of multidrug-resistant bacteria, with some infections now being very difficult to treat or even definitely untreatable, and it is one of the biggest public health challenges of our time. AMR is an increasingly serious threat to global public health that affects hospitals in an epidemic way worldwide. It requires action across all health sectors, government and society. 
Each year in the U.S., at least 2.8 million people get an antibiotic-resistant infection, and more than 35,000 people die. Every year, drug-resistant infections kill 700,000 people worldwide. It has been estimated that, by 2050, 10 million people will die globally every year from drug resistant infections $[60,61]$ if no extensive amendments are made in antibiotic utilization. Fighting this threat is a public health priority that requires a collaborative global approach across sectors to combat this threat [62].

Just to mention two examples, resistance of Klebsiella pneumoniae to a last resort treatment (carbapenem antibiotics) has spread to all regions of the world. K. pneumoniae is a major cause of hospital-acquired infections in newborns and intensive-care unit patients. In some countries, because of resistance, carbapenem antibiotics do not work in more than half of people treated for K. pneumoniae infections. For life-threatening infections caused by Enterobacteriaceae which are resistant to carbapenems, colistin is the last resort treatment. Resistance to colistin has recently been detected in several countries and regions, making infections caused by such bacteria untreatable.

The consequences of bacterial resistance are increased morbidity and mortality of individuals who are affected, burdens on health care systems and unnecessary expenditures which impact neonatal services and the economy of nations.

\section{Economic impact of antibiotic abuse}

There are direct and indirect cost savings by decreasing antibiotic abuse and misuse. These aspects will not be described here; some have been analyzed by others [23,63-65]. In an NICU there was a reduction in costs of $€ 207$ per admitted newborn [23], that would represent $€ 207,000$ in just two NICU's that admit 500 newborns per year each. In the United States overall expenditures on antibiotics for the 6-year period 2010-2015 were estimated in 56 billion dollars $[64,65]$.

\section{Aspects to be considered to decrease mismanagement of antibiotics in the neonatal period}

Recognizing that there is no "one size fits all" approach to optimize antibiotic use for all settings, effective interventions to reduce antibiotic utilization can be designed and implemented in individual NICU's in a collaborative manner by an interdisciplinary team. Assessment of antibiotic consumption is necessary to identify and implement high-yield stewardship targets tailored to the individual center. The neonatal early onset sepsis calculator is an effective tool for antibiotic stewardship in newborns, associated with a reduction of empiric antibiotic treatment for suspected EOBS [19-23,66-68], together with a significant reduction in laboratory investigations and significantly shorter stay in those newborns [23].

\section{Conclusion}

Based on the evidence available in the literature summarized in this review, it is crucial that the attitude in risk-benefit assessment of neonates with suspected sepsis is modified to decrease the risk of serious potential damage caused by the unnecessary abuse or misuse of antibiotics in neonatal medicine. Opportunities exist for antibiotic stewardship among preterm infants [69] and the need for antimicrobial stewardship programs in neonatal medicine in developed and also in low- and middle-income countries has never been more urgent [70]. It is our responsibility in NICU's worldwide to improve the clinical practice of antibiotic therapy.

\section{Acknowledgement}

To the Ibero American Society of Neonatology [SIBEN], a not for profit, public charity organization, and to the efforts of the nurses and neonatologists that collaborate with us in the actions to decrease neonatal abuse of antibiotics in centers and countries of the region.

\section{Conflict of Interest}

No financial or conflict of interest exists for any of the authors.

\section{References}

1. WHO (1985) The Rational Use of Drugs - Report of the Conference of Experts, Nairobi 25-29 November 1985.

2. WHO (2012) The Pursuit of Responsible Use of Medicines: Sharing and Learning from Country Experiences. Switzerland, pp. 78.

3. Ting JY, Roberts A, Sherlock R, Ojah C, Cieslak Z, et al. (2019) Duration of Initial Empirical Antibiotic Therapy and Outcomes in Very Low Birth Weight Infants. Pediatrics 143(3): e20182286.

4. Celiz M, Falco MB, Cantisani MB, Cardetti M, Rodríguez S, et al. (2019) Use of empirical antibiotics early in very low birth weight newborns in SIBEN's neonatal network. SIBEN's annual meeting, October 2019, Quito, Ecuador.

5. Flannery DD, Ross RK, Mukhopadhyay S, Tribble AC, Puopolo KM, et al. (2018) Temporal Trends and Center Variation in Early Antibiotic Use Among Premature Infants JAMA Netw Open 1(1): e180164.

6. Pronovost GN, Hsiao EY (2019) Perinatal Interactions between the Microbiome, Immunity, and Neurodevelopment. Immunity 50(1): 18-36.

7. Zimmermann P, Curtis N (2019) The effect of antibiotics on the composition of the intestinal microbiota - a systematic review. J Infect 79(6): 471-489.

8. Milliken S, Allen RM, Lamont RF (2019) The role of antimicrobial treatment during pregnancy on the neonatal gut microbiome and the development of atopy, asthma, allergy and obesity in childhood. Expert Opin Drug Saf 18(3): 173-185.

9. Coker MO, Hoen AG, Dade E, Lundgren S, Li Z, et al. (2020) Specific class of intrapartum antibiotics relates to maturation of the infant gut microbiota: a prospective cohort study. BJOG 127(2): 217-227.

10. Stokholm J, Schjorring S, Eskildsen CE, Pedersen L, Bischoff AL, et al. (2014) Antibiotic use during pregnancy alters the commensal vaginal microbiota. Clin Microbiol Infect 20(7): 629-635.

11. Mueller NT (2015) The gut microbiome and childhood obesity: connecting the dots. Child Obes 11(3): 227-230.

12. Kuzniewicz MW, Walsh EM, Li S, Fischer A, Escobar GJ (2016) Development and Implementation of an Early-Onset Sepsis Calculator to Guide Antibiotic Management in Late Preterm and Term Neonates. Jt Comm J Qual Patient Saf 42(5): 232-239.

13. Kuzniewicz MW, Puopolo KM, Fischer A, Walsh EM, Li S, et al. (2017) A Quantitative, Risk-Based Approach to the Management of Neonatal Early-Onset Sepsis. JAMA Pediatr 171(4): 365-371. 
14. Achten NB, Dorigo-Zetsma JW, van der Linden PD, van Brakel M, Plötz FB (2018) Sepsis calculator implementation reduces empiric antibiotics for suspected early-onset sepsis. Eur J Pediatr 177(5) :741-746.

15. Flannery DD, Ross RK, Mukhopadhyay S, Tribble AC, Puopolo KM, et al. (2018) Temporal trends and center variation in early antibiotic use among premature infants. JAMA Netw Open 1(1): e180164.

16. Escobar GJ, Puopolo KM, Wi S, Turk BJ, Kuzniewicz MW, et al. (2014) Stratification of risk of early-onset sepsis in newborns $\geq 34$ weeks' gestation. Pediatrics 133(1): 30-36.

17. Lu C, Liu Q, Yuan H, Wang L (2019) Implementation of the Smart Use of Antibiotics Program to Reduce Unnecessary Antibiotic Use in a Neonatal ICU: A Prospective Interrupted Time-Series Study in a Developing Country. Crit Care Med 47(1): e1-e7.

18. Akangire G, Simpson E, Weiner J, Noel-MacDonnell J, Petrikin J, et al. (2020) Implementation of the Neonatal Sepsis Calculator in Early-Onset Sepsis and Maternal Chorioamnionitis. Adv Neonatal Care 20(1): 25-32.

19. Klingenberg C (2018) Early-onset sepsis risk calculator reduces empiric antibiotic use. J Pediatr192: 266-269.

20. Achten NB, Dorigo-Zetsma JW, van der Linden PD, van Brakel M, Plötz FB (2018) Sepsis calculator implementation reduces empiric antibiotics for suspected early-onset sepsis. Eur J Pediatr 177(5): 741-746.

21. Achten NB, Klingenberg C, Benitz WE, Stocker M, Schlapbach LJ, et al. (2019) Association of Use of the Neonatal Early-Onset Sepsis Calculator With Reduction in Antibiotic Therapy and Safety: A Systematic Review and Meta-analysis. JAMA Pediatr173(11): 1032-1040.

22. Strunk T, Buchiboyina A, Sharp M, Nathan E, Doherty D, et al. (2018) Implementation of the Neonatal Sepsis Calculator in an Australian Tertiary Perinatal Centre. Neonatology 113(4): 379-382.

23. Achten NB, Visser DH, Tromp E, Groot W, van Goudoever JB, et al. (2020) Early onset sepsis calculator implementation is associated with reduced healthcare utilization and financial costs in late preterm and term newborns. Eur J Pediatr.

24. Good PI, Hooven TA (2019) Evaluating Newborns at Risk for Early-Onset Sepsis. Pediatr Clin North Am 66(2): 321-331.

25. Sahni M, Franco-Fuenmayor ME, Shattuck K (2019) Management of Late Preterm and Term Neonates exposed to maternal Chorioamnionitis. BMC Pediatrics 19(1): 282

26. Hershkovich-Shporen C (2019) Not all newborns born to mothers with clinical chorioamnionitis need to be treated. J Matern Fetal Neonatal Med 13: 1-6.

27. Sloane AJ, Coleman C, Carola DL, Lafferty MA, Edwards C, et al. (2019) Use of a Modified Early-Onset Sepsis Risk Calculator for Neonates Exposed to Chorioamnionitis. J Pediatr 213: 52-57.

28. Lavoie PM (2019) Rethinking management of neonates at risk of sepsis. Lancet 394(10195): 279-281.

29. Benitz WE, Long SS (2019) The Holy Grail of Ascertainment of EarlyOnset Neonatal Sepsis. J Pediatr 213: 10-12.

30. Brown JVE, Meader N, Cleminson J, McGuire W (2019) C-reactive protein for diagnosing late-onset infection in newborn infants. Cochrane Database Syst Rev 1: CD012126.

31. Brown JVE, Meader N, Wright K, Cleminson J, McGuire W (2020) Assessment of C-Reactive Protein Diagnostic Test Accuracy for LateOnset Infection in Newborn Infants: A Systematic Review and Metaanalysis. JAMA Pediatr.

32. Cantey JB, Bultmann CR (2020) C-Reactive Protein Testing in Late-Onset Neonatal Sepsis: Hazardous Waste. JAMA Pediatr.

33. Puopolo KM, Escobar GJ (2019) Neonatal Sepsis Evaluation: Facing the Certainty of Uncertainty. JAMA Pediatr.

34. Cantey JB, Hersh AL (2019) Antibiotic Stewardship in the Neonatal Intensive Care Unit: Lessons from Oxygen. Pediatrics 143(3).
35. Puopolo KM, Benitz WE, Zaoutis TE, COMMITTEE ON FETUS AND NEWBORN; COMMITTEE ON INFECTIOUS DISEASES (2018) Management of Neonates Born at $\leq 34$ 6/7 Weeks' Gestation With Suspected or Proven Early-Onset Bacterial Sepsis. Pediatrics 142(6): e20182896.

36. Puopolo KM, Benitz WE, Zaoutis TE; COMMITTEE ON FETUS AND NEWBORN; COMMITTEE ON INFECTIOUS DISEASES (2018) Management of Neonates Born at $\geq 350 / 7$ Weeks' Gestation With Suspected or Proven Early-Onset Bacterial Sepsis. Pediatrics 142(6): e20182894.

37. Cantey JB, Sánchez PJ (2011) Prolonged antibiotic therapy for "culturenegative" sepsis in preterm infants: it's time to stop! J Pediatr 159(5): 707-8.

38. Ting JY, Paquette V, Ng K, Lisonkova S, Hait V, et al. (2019) Reduction of Inappropriate Antimicrobial Prescriptions in a Tertiary Neonatal Intensive Care Unit After Antimicrobial Stewardship Care Bundle Implementation. Pediatr Infect Dis J 38(1): 54-59.

39. Schulman J, Benitz WE, Profit J, Lee HC, Dueñas G, et al. (2019) Newborn Antibiotic Exposures and Association With Proven Bloodstream Infection. Pediatrics 144 (5): e20191105.

40. Rueda MS, Calderon-Anyosa R, Gonzales J, Turin CG, Zea-Vera A, et al. (2019) Antibiotic Overuse in Premature Low Birth Weight Infants in a Developing Country. Pediatr Infect Dis J 38(3): 302-307.

41. Cantey JB, Huffman LW, Subramanian A, Marshall AS, Ballard AR, et al. (2017) Antibiotic Exposure and Risk for Death or Bronchopulmonary Dysplasia in Very Low Birth Weight Infants. J Pediatr 181: 289-293.

42. Cantey JB, Pyle AK, Wozniak PS, Hynan LS, Sánchez PJ (2018) Early Antibiotic Exposure and Adverse Outcomes in Preterm, Very Low Birth Weight Infants. J Pediatr 203: 62-67.

43. Flannery DD, Dysart K, Cook A, Greenspan J, Aghai ZH, et al. (2018) Association between early antibiotic exposure and bronchopulmonary dysplasia or death. J Perinatol 38(9): 1227-1234.

44. Ting JY, Roberts A, Sherlock R, Ojah C, Cieslak Z, et al. (2019) Duration of Initial Empirical Antibiotic Therapy and Outcomes in Very Low Birth Weight Infants. Pediatrics 143(3): e20182286.

45. Miller JE, Wu C, Pedersen LH, de Klerk N, Olsen J, et al. (2018) Maternal antibiotic exposure during pregnancy and hospitalization with infection in offspring: a population-based cohort study. Int J Epidemiol 47(2): 561-571.

46. Tremlett H, Bauer KC, Appel-Cresswell S, Finlay BB, Waubant E (2017) The Gut Microbiome in Human Neurological Disease: A Review. Ann Neurol 81: 369-382.

47. Cho I, Blaser MJ (2012) The Human Microbiome: at the interface of health and disease. Nature reviews. Genetics 13(4): 260-270.

48. Cryan JF, O’Riordan KJ, Sandhu K, Peterson V, Dinan TG (2020) The gut microbiome in neurological disorders. Lancet Neurol 19(2): 179-194.

49. Viaud S, Saccheri F, Mignot G, Yamazaki T, Daillère R, et al. (2013) The intestinal microbiota modulates the anticancer immune effects of cyclophosphamide. Science 342(6161): 971-976

50. González Perez G, Hicks AL, Tekieli TM, Radens CM, Williams BL, et al. (2016) Maternal Antibiotic Treatment Impacts Development of the Neonatal Intestinal Microbiome and Antiviral Immunity. J Immunol 196(9): 3768-3779.

51. Cox L, Blaser MG (2015) Antibiotics in early life and obesity. Nat Rev Endocrinol 11(3): 182-190.

52. Bailey LC, Forrest CB, Zhang P, Richards TM, Livshits A, et al. (2014) Association of antibiotics in infancy with early childhood obesity. JAMA Pediatr 168(11): 1063-1069.

53. Dydensborg Sander S, Nybo Andersen AM, Murray JA, Karlstad Ø, Husby S, et al. (2019) Association Between Antibiotics in the First Year of Life and Celiac Disease. Gastroenterology 15(8): 2217-2229. 
54. Ungaro R, Bernstein CN, Gearry R, Hviid A, Kolho KL, et al. (2014) Antibiotics associated with increased risk of new-onset Crohn's disease but not ulcerative colitis: a meta-analysis. Am J Gastroenterol 109(11): 1728-1738.

55. Shaw SY, Blanchard JF, Bernstein CN (2010) Association between the use of antibiotics in the first year of life and pediatric inflammatory bowel disease. Am J Gastroenterol 105(2): 2687-2692.

56. Horton DB, Scott FI, Haynes K, Putt ME, Rose CD ,et al. (2015) Antibiotic exposure and juvenile idiopathic arthritis: a case-control study. Pediatrics 136(2): e333-343.

57. Dik VK, van Oijen MG, Smeets HM, Siersema PD (2016) Frequent use of antibiotics is associated with colorectal cancer risk: results of a nested case-control study. Dig Dis Sci 61(1): 255-264.

58. Nørgaard M, Ehrenstein V, Nielsen RB, Bakketeig LS, Sørensen HT (2012) Maternal use of antibiotics, hospitalisation for infection during pregnancy, and risk of childhood epilepsy: a population-based cohort study. PLoS One 7(1): e30850.

59. Greenberg RG, Chowdhury D, Hansen NI, Smith PB, Stoll BJ, et al. (2019) Prolonged duration of early antibiotic therapy in extremely premature infants. Pediatr Res 85(7): 994-1000.

60. Hutchings MI, Truman AW, Wilkinson B (2019) Antibiotics: past, present and future. Curr Opin Microbiol 51: 72-80.

61.https://www.who.int/antimicrobial-resistance/interagencycoordination-group/final-report/en/

62. https://www.cdc.gov/drugresistance/index.html
63. Suda KJ, Hicks LA, Roberts RM, Hunkler RJ, Danziger LH (2013) A national evaluation of antibiotic expenditures by healthcare setting in the United States, 2009. J Antimicrob Chemother 68(3): 715-718.

64. Suda KJ, Hicks LA, Roberts RM, Hunkler RJ, Matusiak LM, et al. (2018) Antibiotic Expenditures by Medication, Class, and Healthcare Setting in the United States, 2010-2015. Clin Infect Dis 66(2): 185-190.

65. Fischer JE, Ramser M, Fanconi S (2000) Use of antibiotics in PICU and potential savings. Intensive Care Med 26(7): 959-966.

66. Thampi N, Shah PS, Nelson S, Agarwal A, Steinberg M, et al. (2019) Prospective audit and feedback on antibiotic use in neonatal intensive care: a retrospective cohort study. BMC Pediatr 19(1): 105.

67. Schuts EC, Hulscher ME, Mouton JW, Verduin CM, Stuart JWTC, et al. (2016) Current evidence on hospital antimicrobial stewardship objectives: a systematic review and meta-analysis. Lancet Infect Dis 16(7): 847-856.

68. Davey P, Marwick CA, Scott CL, Charani E, McNeil K, et al. (2017) Interventions to improve antibiotic prescribing practices for hospital inpatients. Cochrane Database Syst Rev 2: CD003543.

69. Mukhopadhyay S, Sengupta S, Puopolo KM (2019) Challenges and opportunities for antibiotic stewardship among preterm infants. Arch Dis Child Fetal Neonatal Ed 104: F327-F332.

70. WHO (2019) Antimicrobial stewardship programmes in health-care facilities in low- and middle-income countries. a WHO practical toolkit. Geneva: World Health Organization. 